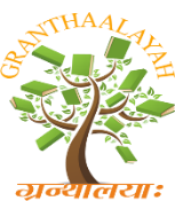

$$
\begin{gathered}
\text { INTERNATIONAL JOURNAL OF RESEARCH } \\
\text { GRANTHAALAYAH } \\
\text { A knowledge Repository }
\end{gathered}
$$

Science

\title{
BENEFICIAL EFFECTS OF MAGNETIC FIELDS ON THE HUMAN HEART A REVIEW OF CURRENT ADVANCES AND PROPOSED MECHANISMS
}

\author{
Abraham A. Embi Bs *1 \\ ${ }^{* 1} 13442$ SW 102 LANE, Miami, Florida, USA 33186, Citizen Scientist
}

\begin{abstract}
The main purpose of this manuscript is to introduce the reader to the present "state of the art" in experimental Low Level Magnetic Fields nerve stimulation approaches to improve chaotic cardiac arrhythmias and muscle contractions. The area addressed in this paper is the transcutaneous application of Low Level Magnetic Fields to a branch of the vagus nerve. Cardiac Arrhythmias such as Atrial Fibrillation, as well as and muscle contractility were reversed by Low Level Transcutaneous Stimulation (LL-TS) of the Auricular Branch of the Vagus Nerve (ABVN). This treatment has been successfully reported in humans as well as in awaken and anesthetized animals such as rodents and dogs. The functional improvement has been attributed to a reversal of cardiac myocytes remodeling (reversal of fibrosis) resulting from experimentally provoking induced rapid heartbeats. The prolonged duration of a tachycardia state induces intracellular accumulation of glycogen leading into fibrosis, this in turn is hypothesized to impede the intercellular electrical communications of the specialized cardiac cells. It also reduces the functional ability of the main pumping chamber (Left Ventricle).
\end{abstract}

Keywords: Hair Biomagnetism; Hair Blood Coagulation; Effect Bio Magnetic Fields; Hair Inhibition Fibrin.

\section{Glossary}

Ablation $=$ Procedure using heat or cold to destroy unwanted tissue.

Atrium $=$ Upper low pressure heart chambers.

Atria $=$ Plural for atrium.

Atrial Fibrillation: Irregular contractions of heart's upper chambers.

ABVN= Auricular Branch of Vagus Nerve.

HPA axis= Hypothalamus-Pituitary-Adrenal axis. Genesis of AF as response to stress.

LLMFs= Low level magnetic fields.

LL-TS= Low Level Transcutaneous Stimulation.

Myocyte $=$ Muscle Cell

Cardiac Myocyte $=$ Heart muscle cells, either from the atria or ventricles.

Cite This Article: Abraham A. Embi Bs. (2018). "BENEFICIAL EFFECTS OF MAGNETIC FIELDS ON THE HUMAN HEART A REVIEW OF CURRENT ADVANCES AND 
PROPOSED MECHANISMS.” International Journal of Research - Granthaalayah, 6(11), 266-271. https://doi.org/10.29121/granthaalayah.v6.i11.2018.1127.

\section{Introduction}

The purpose of this manuscript is to propose a mechanism (based on published data) for the effect on specialized muscle cells (cardiac myocytes) by Low Level Electrical Transcutaneous Stimulation (LL-TS) of the auricular branch of the vagus nerve (ABVN). Glycogen accumulation in atrial myocytes has been hypothesized to induce fibrosis and atrial fibrillation (AF) $(1,2)$. The LL-TS stimulation has been reported to attenuate unwanted interstitial fibrosis and collagen degradation in the atria and ventricle myocytes $(3,4,5)$. Fibrosis has been identified as a factor for arrhythmia, which is a group of conditions where the heartbeat is irregular; it sometimes could lead into severe symptoms requiring medial care. One common arrhythmia AF and its generation have been identified to start in the upper chambers of the heart (atria). Its triggers are multifactorial, such as coronary artery disease and hypertension amongst others; and it may "also occur in the normal heart, a condition known as lone AF" (6). Over the years attempts have been made to control AF by life style modifications (7), pharmacological intervention or aggressive diseased tissue destruction (ablation). Catheter ablation is increasingly offered to patients who suffer from persistent symptoms due to AF, "this recommendation is based on a growing body of evidence illustrating its efficacy compared with antiarrhythmic drug therapy" (8). Atrial remodeling is defined as any change in atrial structure or function that promotes atrial arrhythmias. In the ventricles, remodeling is defined as any change in structure that decreases muscle performance. Preliminary results of LL-TS of the ABVN are encouraging in the atria and left ventricle. The mechanism causing this improvement is unknown at present.

\section{Materials and Methods:}

A literature search was conducted leading to the contents of this manuscript.

\section{Literature Search Findings:}

\section{LL-TS in the Atria (Upper Chambers)}

LL-TS can reverse rapid atrial pacing-induced atrial remodeling and inhibit AF inducibility, suggesting a potential noninvasive treatment of AF.

\section{LL-TS on the Left Ventricle (Main Pumping Chamber)}

Low Level Transcutaneous Stimulation (LLTS) of the auricular branch of the vagus nerve "significantly reduced LV end systolic and end diastolic dimensions. Improved ejection fraction (contractility)", and also "attenuated interstitial fibrosis and collagen degradation in the noninfarcted myocardium when compared with the control group". In both instances, in the atria or left ventricle, LL-TS has contributed to a reversal of the unwanted remodeling.

\section{Glycogen and AF}

The literature search also yielded some interesting findings. We know that by definition myocytes remodeling entails an accumulation of interstitial fibrosis, linked to intramyocyte glycogen accumulation. Also Intracellular glycogen increase in cardiac muscles cells has been hypothesized to be related to the brain Hypothalamic-Pituitary-Adrenal axis (HPA) response to stress (9). 


\section{Acupuncture and AF}

Published data also shows that "acupuncture treatment prevents arrhythmic recurrences after cardioversion in patients with persistent AF. This minimally invasive procedure is safe and well tolerated" $(10,11,12,13)$, Integrated (serial) acupuncture treatments have resulted in AF cessation.

\section{Acupuncture and Hypothalamus Gene Transcription}

Published data also shows a direct effect of acupuncture on hypothalamic DNA methylation. DNA methylation is described as one of several epigenetic (non-genetic mechanisms that cells use to control gene expression), in other words acupuncture places some genes in the "off" position (14).

\section{Association of gene expression with AF}

Published data also shows gene expression with AF. "We examined associations of gene expression with AF in a large community-based cohort. Our study revealed several genes and signaling pathways that are potentially involved in AF-related transcriptional regulation" (15).

\section{Interaction of Fibrinogen with a magnetic source (Protein Coagulation)}

Published data also shows an interaction of fibrinogen with a low level magnetic source. Fibrinogen is a soluble protein present in blood plasma, from which fibrin is produced by the action of the enzyme thrombin. Studies have demonstrated "that the rate of protein coagulation during the formation of fibrin gel under the action of thrombin on fibrinogen decreases $\sim 2$ times in the presence of Low Level emissions by magnetite nanoparticles, and the magnitude of the average fiber mass/length ratio grows" (16).

Brain Cortex Glucose Metabolic Changes are associated with HPA Axis Response to Stressor This most interesting published data links Brain Cortex changes in glucose metabolism with HPA axis response to external stressors, "single electrical stimuli applied to the digital nerves can reduce for a short period the excitability of motor cortex to magnetic stimulation. This occurs at a time when the same stimulus is evoking an excitatory (E2) reflex in the average surface-rectified EMG" (18).

\section{Low Level Magnetic Fields and Fibrin Inhibition}

This published data introduces a microscopic tabletop technique that demonstrates endogenous Low Level Magnetic Fields (LLMFs) biomagnetic tissue crosstalk between human hair and blood. This interaction induces red blood cells (RBCs) agglutination, inhibits fibrin formation (Figure 1) and induces RBCs Rouleaux Formations (19). These findings correlate with the effect of exogenous static magnets as well as pulsating low-level magnetic fields applied to small animals showing to affect blood parameters. There were increases in blood coagulation parameters attributed to the treatments $(20,21)$. Is also in support of the effect of LL-TS of the ABNV. 


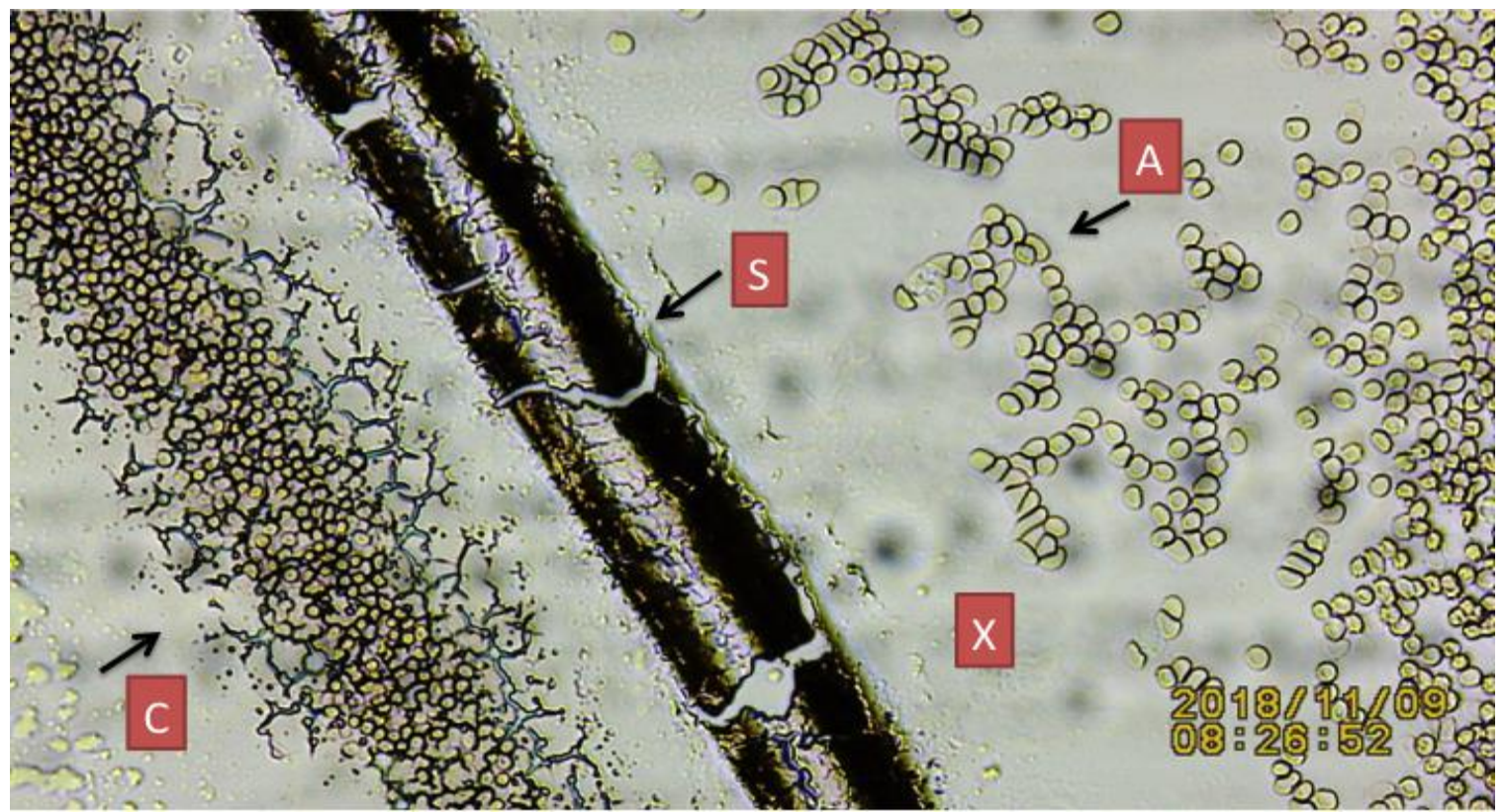

Figure 1: Microphotopraph depicts hair shaft outline. A=RBCs, Agglutinated and in Rouleau

Formation. $\mathrm{S}=$ Hair shaft $\mathrm{C}=$ Coagulated blood, denoting fibrin formation in side void of biomagnetic fields. $\mathrm{X}=$ Diamagnetic zone.

Reprinted from: Embi AA. (2018) HAIR AND BLOOD ENDOGENOUS LOW LEVEL BIOMAGNETIC FIELDS CROSS-TALK EFFECTS ON FIBRIN INHIBITION AND ROULEAU FORMATION. IJRG International Journal of Research - Granthaalayah, 6(11), 200208. https://doi.org/10.5281/zenodo.1845985

\section{Discussion}

This author is aware of the complex interactions between the nervous system and circulatory. The heart being at the center with its blood pumping action and electrical activity; and the brain with its complex signals affecting organ functions. The data presented only scratch the surface of the endogenous molecular, and biomagnetic tissue cross-talk of the human body; nevertheless some conclusions could be inferred by the published findings by this author and others. For example:

1) The literature search also yielded some interesting findings. We know that by definition myocytes remodeling entails an accumulation of interstitial fibrosis, linked to intramyocyte glycogen accumulation. Also Intracellular glycogen increase in cardiac muscles cells has been hypothesized to be related to the brain Hypothalamic-Pituitary-Adrenal Axis (HPA) response to stress (9).

2) Low level Magnetic Fields (LLMFs) are known to be emitted by human tissue such as the hair follicle and shaft. These emissions are as result of metabolism that entails electron transfers creating magnetic fields. Previously published Figure 1 reproduced in this manuscript shows the unilateral LLMFs of a human hair shaft imbedded in a thin blood smear. The displayed image shows the inhibition of fibrin formation by the LLMFs from the right side of the hair shaft. The contralateral side which is void of LLMFs allowed for fibrin formation.

3) The published findings of Transcutaneous Electrical Stimulation of the auricular branch of the vagus nerve (LL-TS) inhibiting or reversing the atrial and ventricular myocytes 
remodeling (reversal of fibrosis) mimics the fibrin inhibition by LLMFs of the hair shaft. This is shown in Figure 1 and other supporting figures are published in (19).

4) The issue of a stimuli to the ear (Auricular Nerve) reversing the cardiac myocytes remodeling, could be explained by a similar approach where the acupuncture like stimulation of the cardiac-related neurons in the auricular point brain medulla evokes cardiovascular inhibition (10). Does a LL-TS nerve stimulation the brain cardiac neurons have the same effect as if applied directly to the cardiac myocytes? The data seems to support the question.

5) The published data also shows a beneficial effect of acupuncture to cardiac myocytes remodeling. The acupuncture data is somewhat isolated to selective patients undergoing arrhythmia conversions via electrical shocks. At present, the data is not reliable enough to be considered as a proposed mechanism for myocytes fibrin inhibition.

\section{Summary}

The printed body of knowledge search in the area of the effect of glycogen, fibrosis and LL-TS on cardiac arrhythmias yielded the references cited in this manuscript.

It can be stated that the success of LL-TS of the ABVN on reversing atrial and ventricular myocytes remodeling is caused by an inhibition in fibrin formation. That the underlaying common denominator mechanism of the inhibition appears to involve protein coagulation.

\section{References}

[1] Embi, A.A., Scherlag, B. J., \& Ritchey, J. W. (2014). Glycogen and the propensity for atrial fibrillation: intrinsic anatomic differences in glycogen in the left and right atria in the goat heart. North American journal of medical sciences, 6(10), 510-5. PMC4215488

[2] Ling Zhang, Bing Huang, Benjamin J Scherlag, Jerry W Ritchey, Abraham A Embi, Jialu Hu, Yuemei Hou, Sunny S Po (2015) Structural changes in the progression of atrial fibrillation: potential role of glycogen and fibrosis as perpetuating factors Int J Clin Exp Pathol. 2015; 8(2): 1712-1718. PMC4396299

[3] 3) Yu L, Scherlag BJ, Li S, Fan Y, Dyer J, Male S, Varma V, Sha Y, Stavrakis S, Po SS. (2013) Low-level transcutaneous electrical stimulation of the auricular branch of the vagus nerve: a noninvasive approach to treat the initial phase of atrial fibrillation. ;10(3):428-35. PMID: 23183191

[4] 4) Wang Z, Yu L, Wang S, Huang B, Liao K, Saren G, Tan T, Jiang H. (2014) Chronic intermittent low-level transcutaneous electrical stimulation of auricular branch of vagus nerve improves left ventricular remodeling in conscious dogs with healed myocardial infarction. Circ Heart Fail. 2014 Nov;7(6):1014-21. PMID:25332149

[5] Wang Z, Yu L, Huang B, Wang S, Liao K, Saren G, Zhou X, Jiang H. (2015) Low-level transcutaneous electrical stimulation of the auricular branch of vagus nerve ameliorates left ventricular remodeling and dysfunction by downregulation of matrix metalloproteinase 9 and transforming growth factor $\beta 1$. J Cardiovasc Pharmacol. 2015 Apr;65(4):342-8. doi: 10.1097/FJC.0000000000000201. PMID:25502306

[6] Sánchez-Quintana, D., López-Mínguez, J. R., Pizarro, G., Murillo, M., \& Cabrera, J. A. (2012). Triggers and anatomical substrates in the genesis and perpetuation of atrial fibrillation. Current cardiology reviews, 8(4), 310-26. PMC3492815 
[7] Abdul-Aziz, Ahmad A. et al. (2018) Lifestyle Therapy for the Management of Atrial Fibrillation American Journal of Cardiology, Volume 121, Issue 9, 1112 - 1117

[8] Kirchhof, P., \& Calkins, H. (2016). Catheter ablation in patients with persistent atrial fibrillation. European heart journal, 38(1), 20-26.

[9] Embi, A. A., \& Scherlag, B. J. (2014). An endocrine hypothesis for the genesis of atrial fibrillation: the hypothalamic-pituitary-adrenal axis response to stress and glycogen accumulation in atrial tissues. North American journal of medical sciences, 6(11), 586-90. PMC4264295.

[10] Gao, X.Y., Li, Y.H., Liu, K., Rong, P.J., Ben, H., Li, L., Zhu, B., Zhang, S.P. 2011. Acupuncturelike stimulation at auricular point Heart evokes cardiovascular inhibition via activating the cardiacrelated neurons in the nucleus tractus solitarius. Brain Research 1397, 19-27.

[11] Alberto Lomuscio M.D. Sebastiano Belletti M.D. Pier Maria Battezzati M.D. Federico Lombardi M.D., F.E.S.C. (2011) Efficacy of Acupuncture in Preventing Atrial Fibrillation Recurrences After Electrical Cardioversion. J Cardiovasc Electrophysiol, Vol. 22, pp. 241-247.

[12] Jonkman FA, Jonkman-Buidin ML. (2013) Integrated approach to treatment-resistant atrial fibrillation: additional value of acupuncture. Acupunct Med. 2013 Sep;31(3):327-30. PMID:23884291

[13] Dilber D, Čerkez-Habek J, Barić H, Gradišer M. (2015) Atrial fibrillation cardioversion following acupuncture. Saudi Med J. 2015 Nov;36(11):1351-3. doi: 10.15537/smj.2015.11.12891. PMID:26593171

[14] Cui P, Ma T, Tamadon A, Han S, Li B, Chen Z, An X, Shao LR, Wang Y Feng Y. (2018) Hypothalamic DNA methylation in rats with dihydrotestosterone-induced polycystic ovary syndrome: effects of low-frequency electro-acupuncture. Exp Physiol. 2018 Sep 11. doi: 10.1113/EP087163. [Epub ahead of print] PMID:30204276

[15] Lin H, Yin X, Lunetta KL, Dupuis J, McManus DD, Lubitz SA, et al. (2014) Whole Blood Gene Expression and Atrial Fibrillation: The Framingham Heart Study. PLoS ONE 9(5): e96794.

[16] A.V. Bychkova, O.N. Sorokina, A.L. Kovarski, A.B. Shapiro, V.B. Leonova, M.A. Rozenfel'd, (2010) Interaction of fibrinogen with magnetite nanoparticles. Biophysics. Vol. 55, No. 4, pp. 544549.

[17] Simone Kern, Terrence R. Oakes, Charles K. Stone, Emelia M. McAuliff, Clemens Kirschbaum, and Richard J. Davidson. (2008). Glucose metabolic changes in the prefrontal cortex are associated with HPA axis response to a psychosocial stressor. Psychoneuroendocrinology. 2008 May; 33(4): 517-529.

[18] Maertens de Noordhout A, Rothwell JC, Day BL, Dressler D, Nakashima K, Thompson PD, Marsden CD. (1992) Effect of digital nerve stimuli on responses to electrical or magnetic stimulation of the human brain. J Physiol. 1992 Feb; 447:535-48.

[19] Embi AA. (2018) HAIR AND BLOOD ENDOGENOUS LOW LEVEL BIOMAGNETIC FIELDS CROSS-TALK EFFECTS ON FIBRIN INHIBITION AND ROULEAU FORMATION, IJGR (in print).

[20] Goraca A, Michalska M. (2005). [The effect of low magnetic field on select parameters of blood coagulation]. Pol Merkur Lekarski. (110):148-51.

[21] Dhahir T. Ahmad (2011) Effects of Low Frequency Pulsed Magnetic Field on Blood Clotting Time in Male Rabbits. Diyala Journal of Medicine. Vol. 1, Issue 2: pp. 56-63.

*Corresponding author.

E-mail address: embi21@ att.net 